\title{
3D Air-Ground Channel Model Reconstruction Based on the Experimental Data and Q-D Method
}

\author{
Alexander MALTSEV ${ }^{\mathrm{a}, \mathrm{b}}$, Olesya BOLKHOVSKAYA ${ }^{\mathrm{b}, 1}$, Vadim SERGEEV ${ }^{\mathrm{b}}$, Anton \\ ELOKHIN $^{\mathrm{b}}$ and Alexander KOKAREV ${ }^{\mathrm{b}}$

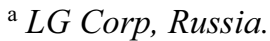 \\ ${ }^{\mathrm{b}}$ University of Nizhny Novgorod, Russia
}

\begin{abstract}
This paper proposes to use the Q-D (Quasi-Deterministic) method for reconstructing the angular and frequency characteristics of the 3D Air-to-Ground (A-G) channel from the available experimental data. This method allows to expand the applicability of tapped delay line (TDL) channel models for performance investigation of the aviation radio systems with directional antennas and multielement antenna arrays. The developed 3D A-G channel models also give opportunity to take into account Doppler spread impact on the communication system performance.
\end{abstract}

Keywords. 3D Air-Ground channel, CIR, AoA, AoD, Doppler spread

\section{Introduction}

In recent years, it has been observed the very rapid development of unmanned aerial vehicles (UAV) technologies and their increasing use in various military and civil applications. Therefore, the number of works devoted to the study of characteristics and models of radio channels that provide UAV flight control and data transfer between them and ground stations has increased dramatically. Currently, many research organizations and standardization committees, such as [1], [2], are engaged in building radio communication networks with UAVs.

The developed analytical models [3]-[8] do not always describe the real behavior of the Air-to-Ground (A-G) channels due to insufficiently realistic assumptions. Therefore, in recent years, quite intensive experimental studies of radio communication channels for UAVs have been conducted to build their empirical models [9]-[11].

The most detail experimental studies of the A-G channel for UAV were conducted in 2013-2017 as part of a project funded by the NASA J. Glenn research center [12][15]. During the implementation of this project, a whole set of experimental measurements of the A-G radio channels in two frequency bands (L-band from 900$977 \mathrm{GHz}$, and C-band from 5030-5091 GHz) was carried out in various scenarios: over water, in hilly and mountainous areas, in suburban and urban environments. Based on the gathered experimental data, evaluation of path losses, power delay profiles, K-

${ }^{1}$ Corresponding Author: Olesya Bolkhovskaya, University of Nizhny Novgorod, Gagarina ave,23, 603109 Nizhny Novgorod, Russia; E-mail:obol@rf.unn.ru 
factors, spatial correlation coefficients was made for all considered scenarios, and TDL channel models were developed.

However, for practical applications, it is desirable to have more detailed 3D A-G channel models that adequately describe the spatial, temporal, angle and frequency characteristics of the channel, and allow investigation of the modern aviation radio systems with directional and multi-element antennas.

In this paper, we, inspired by experimental data available from [12]-[15], propose to apply the Q-D method [16]-[17] to reconstruct the angular and Doppler spread characteristics of the rays. The key benefit of the Q-D approach compared to pure statistical channel models is its inherent support for spatial consistency. In this approach, the deterministic part of the channel impulse response (so called, deterministic rays) accurately takes into account the positions of the transmitter TX, receiver RX and a few strongest reflectors, always existing in considered scenario. The other part of the channel impulse response represents a number of relatively week random reflections (so called, random rays). Simulating moving objects, the Q-D channel model can accurately reproduce fading and Doppler effects, observed in real measurements [16]. This is not possible with a purely statistical model. At the same time, compared to the fully deterministic ray-tracing method, the Q-D approach has much less computational complexity.

It should be mentioned, that the Q-D approach, used for recovering the missing parameters for building 3D A-G channel models, also allows us to expand the scope of application of the developed models to the different heights of the transmitter and receiver locations.

It is interesting to note, that the Q-D method was initially introduced for development of 3D channel models for the millimeter wave bands [16] and then justified in a number of experimental measurements [17-18]. In the present paper, we apply the Q-D approach to build 3D A-G channel models for the centimeter wave Cband, because the experimentally measured characteristics of the A-G channel for Cband [12-15] are very similar to the characteristics of the millimeter wave $57-64 \mathrm{GHz}$ channel for the Open Area outdoor hotspot scenario [17]. The physical background for this similarity is approximately the same ratio of the wavelength $\lambda$ to the typical scale of the environment $d$ in both bands. Indeed, for millimeter wave channel, this ratio is about $\lambda / \mathrm{d}=5 \mathrm{~mm} / 100-300 \mathrm{~m}$, and for the C-band $\lambda / \mathrm{d}=6 \mathrm{~cm} / 1-3 \mathrm{~km}$. It results in a $1: 10$ scale similarity of the $3 \mathrm{D}$ channel impulse responses for these channels and justifies the application of the Q-D methodology for the C-band in the considered case.

\section{Problem Statement}

The Q-D method for recovering the unknown parameters of the channel rays exploits the combination of experimental data and some additional information about the TX and RX positions, antenna heights, properties of ground or water underlying surfaces, roughness coefficients, location of surrounding reflectors, etc. All these information should be specified in the description of the scenario. The 3D A-G channel model development is based on the experimental data provided in the set of papers [12]-[15]. The scheme of the typical experiment from these papers is shown in Figure 1. A scheme of the experimental measurements of the Air-to-Ground (A-D) radio channel characteristics [12]-[15]. 
The 10-watt sounding signal transmitter was located at the ground station (GS) with a nearly omnidirectional in azimuth antenna on height of 20 meters above ground level, while the receiver was located on an aircraft flying at an altitude of 800 to 1200 meters at various distances of 2-50 km from the GS. The Q-D approach for channel modeling is built on the representation of the channel impulse response comprised of a few quasi-deterministic strong rays (D-rays) and a number of relatively weak random rays (R-rays), originating from the surrounding objects reflections.

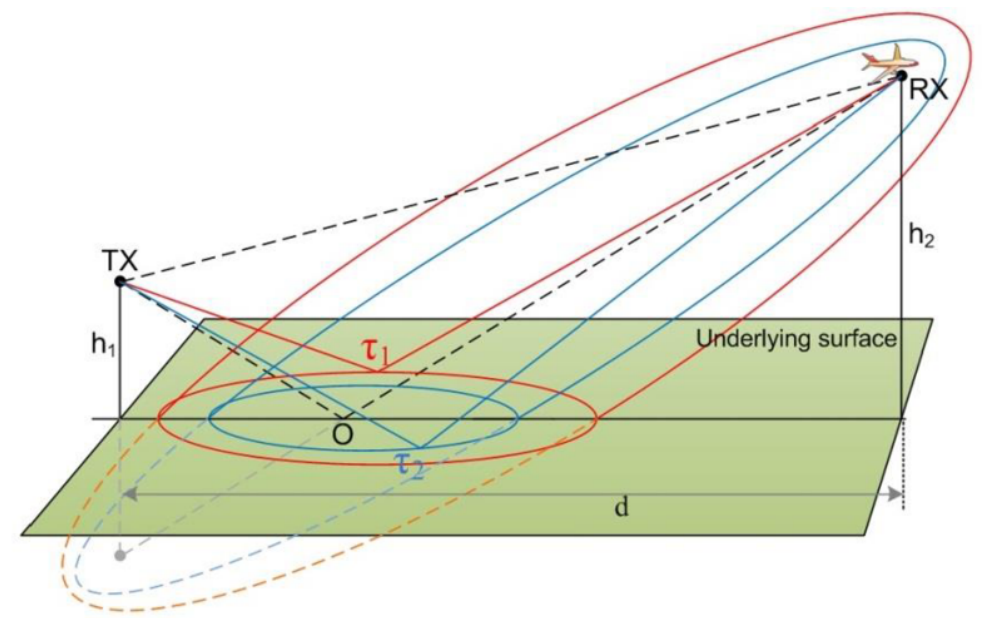

Figure 1. A scheme of the experimental measurements of the Air-to-Ground (A-D) radio channel characteristics [12]-[15].

The set of D-rays for open area scenario includes only two rays: direct LOS (lineof-sight) ray and underlying surface reflected ray (see Figure 1). The characteristics of these both D-rays for the 3D A-D channel model may be calculated form system geometry, properties of ground surface and available experimental data [12]-[15].

The R-rays, in accordance with the Q-D methodology [16]-[17], may be generated in two different ways: statistically from the pre-defined power-delay profile or as deterministic reflections from the random surrounding objects. In this paper we use statistical characteristics of the R-rays defined in [12]-[15] and, for recovering unknown angular and frequency ray parameters, convert them into reflections from the random surrounding objects as it will be shown below.

The A-G channel models, developed in [12]-[15], introduce four types of the multipath channel rays (see Figure 2):

1. Direct LOS D-ray indicated by the wave vectors $\mathbf{r}_{\mathbf{T X}, \mathbf{1}}$ and $\mathbf{r}_{\mathbf{R X}, \mathbf{1}}$.

2. NLOS (Non-line-of-sight) D-ray reflected from the underlying surface and indicated by the wave vectors $\mathbf{r}_{\mathbf{T X}, 2}$ and $\mathbf{r}_{\mathbf{R X}, 2}$.

3. R-rays that have time delays exceeding the delay of the ground reflected NLOS D-ray. These R-rays indicated by the wave vectors $\mathbf{r}_{\mathbf{T X}, \mathbf{i}}$ and $\mathbf{r}_{\mathbf{R X}, \mathbf{i} \text {. }}$

4. R-rays that have time delays less than the delay of the ground reflected NLOS D-ray. These R-rays indicated by the wave vectors $\mathbf{r} \mathbf{T X}, \mathbf{j}$ and $\mathbf{r} \mathbf{R X}, \mathbf{j}$.

Solving the problem of the $3 \mathrm{D}$ A-G channel reconstruction, it is necessary to find (generate) the values of angles of departure (AoD), angles of arrival (AoA) and frequency shifts for each of the above types of the channel rays. 


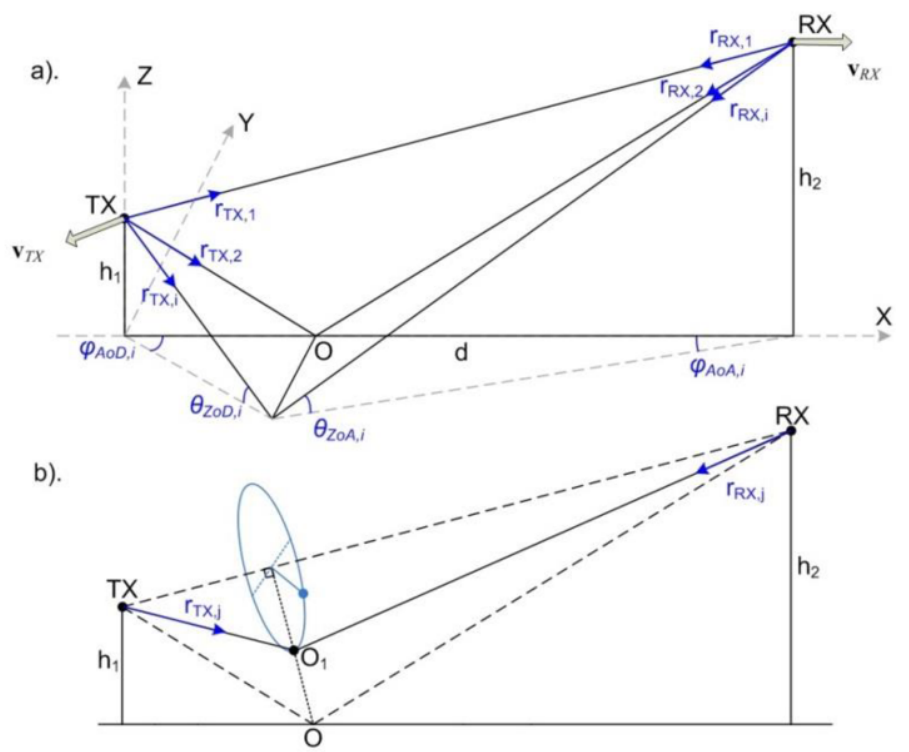

Figure 2. Four types of the A-D multipath channel model rays: LOS D-ray, NLOS D-ray reflected from the underlying surface, R-rays of type 3 and R-ray of type 4 with different delays.

\section{Channel Angular Parameters Reconstruction Algorithms}

The Q-D approach, used by the authors in this paper, involves calculating the AoD and AoA based on defining the position of channel reflectors in the space between the transmitter and receiver [16]-[17]. This task is solved in two steps: the first one is calculating the possible position of channel reflectors for each of the ray based on the known delays of the rays in the channel impulse response, and, the second one is calculating the AoD and AoA based on the relative locations of the TX, RX and the defined channel reflectors.

We consider the reconstruction process for the departure and arrival angles for each of the four types of rays. For the direct LOS D-ray and the NLOS D-ray reflected from the underlying surface the departure and arrival angles are calculated directly from the coordinates of the TX and RX. Note, that for these D-rays, in the azimuth plane, the angles of departure (AzoD) and the angles of arrival (AzoA) can be set to zero, without loss of generality, by selecting the appropriate coordinate system (see Figure 2a).

Two assumptions have been made for the $3^{\text {rd }}$ type R-rays that delay exceeds the delay of the ground reflected NLOS D-ray. First one, it is assumed that the trajectory of each R-ray is determined by a single reflection from some GS surrounding object, and second, this object is located near the earth's surface. Under these assumptions, the coordinates of the reflector generating this type of a R-ray lies on the underlying surface on some ellipse, which is the intersection of the corresponding ellipsoid of equal delays. For example, Figure 1 shows a scheme for calculating the position of channel reflectors for the $3^{\text {rd }}$ type R-rays with delays $\tau_{1}$ and $\tau_{2}$.

Following these assumptions, at the first step for each $3^{\text {rd }}$ type R-ray the corresponding its known delay ellipse is constructed on the ground surface. Because, 
the delay of the reflected ray does not give any reason to prefer a particular point on the ellipse, as the final position of the reflector, the choice of this point is made randomly. The point on the ellipse that is visible from the reflection point O of the NLOS D-ray at the random angle (uniformly distributed from 0 to $2 \pi$ ) is selected as the location of the reflector generating this $3^{\text {rd }}$ type R-ray.

For the 4th type R-rays those delays less the delay of the ground reflected NLOS D-ray, the reflecting object cannot lie on the underlying surface, since this D-ray has the minimum delay of all the rays reflected from this surface. Therefore, for the $4^{\text {th }}$ type R-rays, the reflection point must lie above the underlying surface (earth or water). For example, it can be a reflection from a high building or from another high object that rises above the average ground level. The construction of the $4^{\text {th }}$ type R-ray path, according to this assumption, is illustrated in Figure $2 \mathrm{~b}$. At the first step, at the height of the triangle O-RX-TX, drawn from point $\mathrm{O}$, the point $\mathrm{O} 1$ corresponding to the R-ray delay is selected. Then, at the second step, the final point of the $4^{\text {th }}$ type R-ray reflection is determined by rotating the point $\mathrm{O}_{1}$ in space around the LOS direction TX$\mathrm{RX}$ at the random angle in the range from $-\pi / 2$ to $\pi / 2$. Since the rotation occurs around the line of sight, the final R-ray trajectory does not change the delay of the ray, but it adds additional variability to the values of the AoD and AoA of the 4rd type Rray.

After calculating the coordinates of the reflection points for each of the R-rays from the channel impulse response (CIR), azimuth and elevation AoD and AoA in the global coordinate system are calculated using simple geometric constructions (see Figure 2).

\section{Doppler Shift Calculation}

The angular characteristics of the 3D A-G channel model, found by using the algorithms described in the previous section, make it possible to calculate the Doppler shifts for each ray in the CIR. Since the proposed reconstruction procedure makes it possible to set the AoD and AoA for each ray, the Doppler frequency offset can be easy calculated for each channel ray by setting the direction and speed of the transmitter and receiver relatively to the global coordinate system (see Figure 2). Therefore, in the considered approach, the Doppler spread of the transmitted signal spectrum is modeled as a set of Doppler shifts to the signal carrier frequency for each of the channel ray [17]-[18]. In the case of fixed channel reflectors and moving TX and / or RX, for each channel ray the Doppler frequency shift can be represented as the sum of frequency shifts before and after reflection (see Figure 2). So for the i-th ray:

$$
f_{d, i}=\frac{f_{0}}{c}\left(\mathbf{v}_{T X} \cdot \mathbf{r}_{T X, i}+\mathbf{v}_{R X} \cdot \mathbf{r}_{R X, i}\right),
$$

where $\mathbf{V}_{\mathbf{T X}}$ and $\mathbf{V}_{\mathbf{R X}}$ are the velocity vectors of the transmitter and receiver, respectively, and $\mathbf{r}_{\mathbf{T X}, \mathbf{i}}$ and $\mathbf{r}_{\mathbf{R X}, \mathbf{i}}$ are the unit wave vectors of the ray path for the TX and RX, respectively, $\mathrm{f}_{0}$ is the carrier frequency, $c$ is the speed of light. 


\section{Simulation Results}

In the paper by the using the experimental data and characteristics of the TDL channel models given in [12]-[15], as well as on the basis of the developed Q-D methodology, a software model of the 3D A-G channel was implemented. At the same time, the approach to the channel modeling was slightly changed compared to the original TDL models from [12]-[15] in order to bring the methodology closer to the standards adopted for the development of wireless communication technologies by IEEE and 3GPP.

The system level simulation (SLS) platform for the 3D A-G channel model environment was created in the Matlab and $\mathrm{C}++$. The software implementation includes procedures for the CIR generation, the AoD and AoA calculation for individual rays, and the ray Doppler shifts determination. The CIR generation involves calculating the power of the LOS D-ray, the NLOS mirror-reflected D-ray, and several R-rays with time delays both greater and less than the NLOS D-ray delay. For LOS and NLOS D- rays, the attenuation of their power in space is taken into account, as well as additional path losses due to the weather factors and vegetation. The R-rays generation was performed in accordance with statistical distributions of their delays and amplitudes given in [12]-[15]. After obtaining the CIRs and using the reconstruction algorithms described in the previous sections of this paper, the angular characteristics and Doppler shifts of all the rays are calculated.

To check the adequacy of the developed 3D A-G channel models, ensembles of the CIRs were generated depending on the horizontal distance between the transmitting GS and the receiver located on the aircraft. Examples of these ensembles for flight scenarios "over-sea" (the GS height $20 \mathrm{~m}$ and aircraft altitude $800 \mathrm{~m}$ ) and "over nearurban environment" (the GS height $20 \mathrm{~m}$ and aircraft altitude $562 \mathrm{~m}$ ) are shown in Figure $3 \mathrm{a}$ and Figure $3 \mathrm{~b}$, respectively. More detail comparison of these SLS results with experimental results, represented in [12] and [14], shows that the developed 3D A-G channel model allows us to adequately reproduce the statistics of the real CIRs.
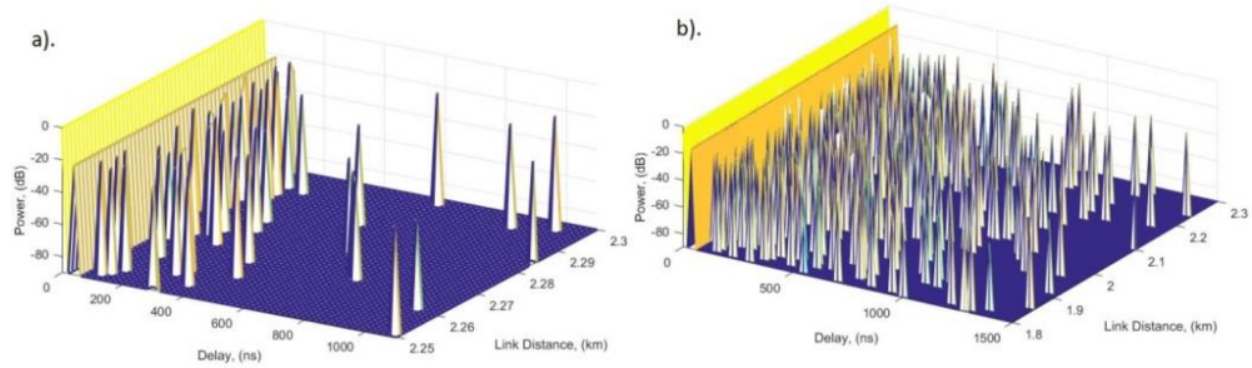

Figure 3. Ensembles of the CIRs depending on the distance between the transmitter and receiver: A-flight scenario "over-sea"; B-flight scenario "over near-urban environment".

The histograms of the AoA and AoD distributions in the azimuth and elevation planes for the scenarios "over-sea" and "over near-urban environment", obtained by using the reconstruction algorithms, described in the previous sections of the paper, shown in Figure 4 and Figure 5, respectively. 
a).

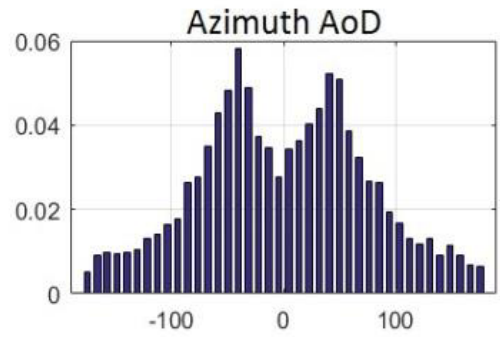

c).

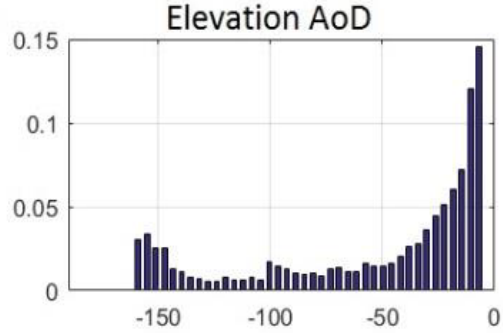

b).

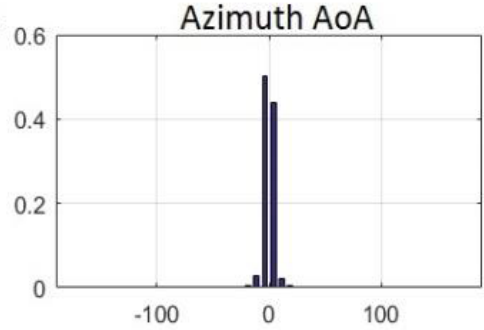

d).

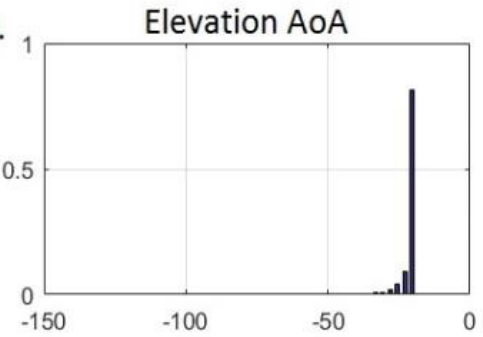

Figure 4. Histograms of the 3D A-G channel model ray angles distribution in the "over-sea" scenario: azimuth AoD and AoA on top, elevation AoD and AoA on bottom. The TX height is $20 \mathrm{~m}$, the RX height is $800 \mathrm{~m}$, the horizontal distance between the TX and RX is $2260 \mathrm{~m}$.

a).

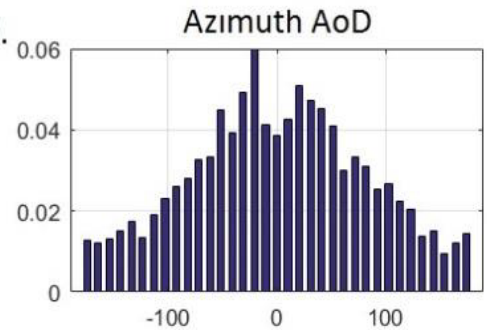

c).

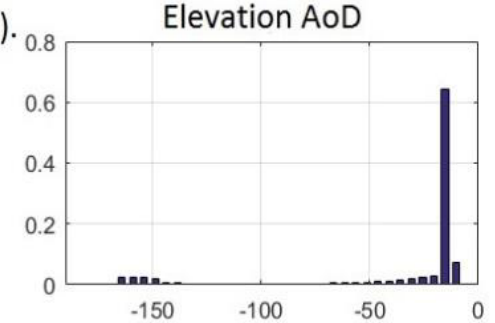

b).

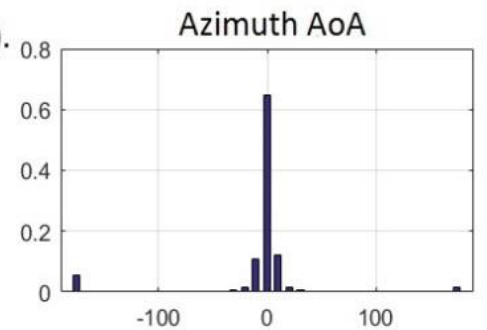

d).

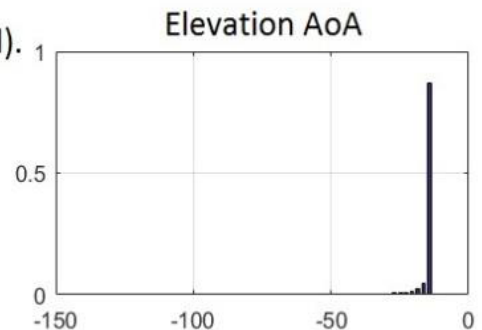

Figure 5. Histograms of the 3D A-G channel model ray angles distribution in the "over near-urban environment" scenario: azimuth AoD and AoA on top, elevation AoD and AoA on bottom. The TX height is $20 \mathrm{~m}$, the RX height is $562 \mathrm{~m}$, the horizontal distance between the TX and RX is $2260 \mathrm{~m}$.

It can be seen that for the TX, located at the GS near the ground, the AoD distributions in azimuth and elevation planes are much wider for both scenarios than for the RX located at the high altitude aircraft. This is due to the fact that the bulk of 
the random R-rays in the A-G channel are obtained as a result of reflection from the GS surrounding objects on the ground surface. At the same time, the angular size of the reflection area is significantly smaller when observed from the aircraft, than when observed from the GS.

It is interesting to note that for both scenarios, the distributions of the azimuth and elevation AoAs on the RX (aircraft) have approximately the same narrow width. The angular distributions of the departure rays for different scenarios turn out to be significantly different, which is probably determined by the various configurations of objects surrounding the transmitting GS.

\section{Conclusion}

In this paper, we have introduced a Q-D method for reconstructing the angular and frequency characteristics of the 3D A-D channel models based on the empirical TDL channel models [12]-[15]. The SLS platform for modeling all considered scenarios and environments was created in the Matlab. Simulations have shown that the developed 3D A-G channel model adequately reproduces the statistics of the real CIRs and provides physically reasonable AoD and AoA distributions and Doppler spreads. Thus, the authors believe that this model is useful for characteristics evaluation of modern UAV and aircraft communication systems with directional and multi-element antennas. As the further steps in this work, the authors see in introduction of a more detail cluster structure of channel rays (including flash rays F-rays), which observed in experiments, and upgrade the SLS platform for modeling both the short and long term dynamical changes of the A-G channel.

\section{References}

[1] Radio Technical Commission for Aeronautics (RTCA), SC-228, "Minimum Operational Performance Standards for Unmanned Aircraft Systems", 2013, http://www.rtca.org/content/sc-228.

[2] National Aeronautics and Space Administration, 2014, available online at http://www.aeronautics.nasa.gov/isrp/uas/index. html.

[3] E. Haas, Aeronautical channel modeling, IEEE Transactioncs on vehicular technology, 2 (2002), 254264.

[4] J. Sykora, Tapped delay line model of linear randomly time-variant WSSUS channel, Electronics Letters, 36 (2000), 1656-1657.

[5] K. Mammasis, E. Pfann, R. W. Stewart and G. Freeland, Three-dimensional channel modelling using spherical statistics for smart antennas, Electronics Letters, 44 ( 2008), 769-774.

[6] A. Al-Hourani, S. Kandeepan and A. Jamalipour, Modeling Air-to-Ground Path Loss for Low Altitude Platforms in Urban Environments, Proc. IEEE Global Commun. Conf., (2014), 2898-2904.

[7] M.Wentz and M. Stojanovic, A MIMO Radio Channel Model for Low-Altitude Air-to-Ground Communication Systems, Proc. IEEE Veh. Technol Conf. (VTC-Fall'15), (2015), 1-6.

[8] S. Gulfam, S. Nawaz, M. Patwary and M. Maguid, On the Spatial Characterization of 3-D Air-to-Ground Radio Communication Channels, Proc. IEEE Int. Conf. Commun. London, (2015), 2924-2930.

[9] M. Simunek, F.P. Fontan and P. Pechac, The UAV Low Elevation Propagation Channel in Urban Areas: Statistical Analysis and Time-Series Generator, IEEE Trans. Antennas Propag., 61 (2013), 3850-3858.

[10] E. Yanmaz, S. Hayat, J. Scherer and C. Bettstetter, Experimental Performance Analysis of Two-Hop Aerial 802.11 Networks, Proc. IEEE Wireless Commun. Netw. Conf., (2014), 3118-312.

[11] W. Khawaja, I. Guvenc and D. Matolak, UWB Channel Sounding and Modeling for UAV Air-toGround Propagation Channels, Proc. IEEE Global Commun. Conf. (GLOBECOM'16), (2016), 1-7.

[12] D.W. Matolak and R. Sun, Air-Ground Channel Characterization for Unmanned Aircraft Systems-Part I: Methods Measurements and Models for Over-Water Settings, IEEE Trans. Veh. Technol, 66 (2017), $26-44$. 
[13] R. Sun and D.W. Matolak, Air-Ground Channel Characterization for Unmanned Aircraft Systems-Part II: Hilly and Mountainous Settings, IEEE Trans. Veh. Technol., 66 (2017), 1913-1925.

[14] D.W. Matolak and R. Sun, Air-Ground Channel Characterization for Unmanned Aircraft Systems-Part III: The Suburban and Near-Urban Environments, IEEE Trans. Veh. Technol., 66 (2017), 6607-6618.

[15] R.Sun and D.W. Matolak, Air-Ground Channel Characterization for Unmanned Aircraft Systems PartIV: Airframe Shadowing, IEEE Trans. Veh. Technol, 66 (2017), 7643-7652.

[16] A. Maltsev et al., Quasi-deterministic approach to mmWave channel modeling in a non-stationary environment, 2014 IEEE Globecom Workshops (GC Wkshps), (2014), 966-971.

[17] A. Maltsev et al. Channel Models for IEEE 802.11ay, Doc. IEEE 802.11-2015/1150r9 (2017).

[18] J. Wang, C. Gentile, P. B. Papazian, J. Choi and J. Senic, Quasi-Deterministic Model for Doppler Spread in Millimeter-Wave Communication Systems,IEEE Antennas and Wireless Propagation Letters, 16 (2017). 